\title{
Design of control systems for fermenters at the Molecular Immunology Center
}

\section{Waldo Hernández Sánchez ${ }^{1}$, Aneisys González Gonzalez ${ }^{2}$, Daniel Darias Zayas ${ }^{3}$, Daniel Gongora Morales ${ }^{4}$, Gismell Garcia Garcia ${ }^{5}$, Ronny O. Pacheco ${ }^{6}$}

\author{
1,2,3,4,5,6 Centro de Inmunología Molecular, Calle 216 esq 15 Siboney Playa La Habana, Cuba. \\ Email: aneisys@cim.sld.cu, danield@cim.sld.cu,danielg@cim.sld.cu,gismell@cim.sld.cu,ronny@cim.sld.cu
}

Received: November $24^{\text {th }}, 2017$.

Accepted: December $05^{\text {th }}, 2017$.

Published: December $13^{\text {th }}, 2017$.

Copyright $\odot 2016$ by authors and Institute of Technology Galileo of Amazon (ITEGAM). This work is licensed under the Creative Commons Attribution International License (CC BY 4.0).

http://creativecommons.org/licenses/by/4.0/ (c) (1) (2) Opea Actes:

\begin{abstract}
The culture of mammals cells represents the main productive link of the Molecular Immunology Center (CIM) and the fermenters represent the heart of this productive and investigative processes. For the inability to grow in new fermentation equipment due to its high costs and services, we have been given the task of recovering equipment already discontinued due to problems in its control systems. This work is based on the control system implemented in the TechFors Fermenter situate on CIM's pilot plant, whose main problems were in the expiration in its control system, errors in sequences of operation and measurement of variables, as well as lack of Technical support for the obsolescence of technology. To develop the re-automation of this equipment was used a PLC of the manufacturer Siemens to implement the control system and an industrial PC to carry out the monitoring and supervision of the system. STEP7 and WinCC Flexible softwares were used to develop programming and adjustment of all control loops, operations and equipment supervision. As a main result a new control system was designed and implemented in a fermenter, demonstrating that it is possible to rescue those already abandoned systems of fermentation over the years, giving them efficient and durable controls again.
\end{abstract}

Keywords: Fermenter; PLC; Software.

\section{RESUMEN}

El cultivo de células de mamíferos o superiores representa el principal eslabón productivo del Centro de Inmunología Molecular (CIM) y los fermentadores constituyen el corazón dentro de este engranaje de procesos productivos e investigativos. Por la imposibilidad de crecer en nuevos equipos de fermentación por sus elevados costos y servicios, nos hemos dado a la tarea de recuperar equipos ya descontinuados por problemas en sus sistemas de control. Este trabajo se basa en el sistema de control implementado en el Fermentador TechFors de 14Litros de la planta piloto del CIM, cuyos principales problemas estaban en la caducidad de su sistema de control, errores en secuencias de funcionamiento y medición de variables, así como falta de soporte técnico por la obsolescencia de la tecnología. Para desarrollar la re-automatización de este equipo se utilizó un PLC del fabricante Siemens para implementar el sistema de control y una PC industrial para realizar el monitoreo y supervisión del sistema. Se utilizaron los softwares de control STEP7 y el WinCCFlexible para desarrollar la programación y ajuste de todos los lazos de control, las operaciones y la supervisión del equipo. Como resultado principal se diseñó e implemento un nuevo sistema automatizado en el fermentador, demostrando que es posible el rescate de aquellos sistemas de fermentación ya caducos por el paso de los años, dotándolos nuevamente de controles eficientes y duraderos.

Palabras Clave: Fermentador; PLC; Software. 


\section{INTRODUCCIÓN}

La biotecnología en Cuba ha tenido un amplio desarrollo en las últimas décadas, contando ya con varias instituciones que se dedican a la ingeniería genética, la investigación y a la producción de biofármacos, aportando así cuantiosos ingresos al país cada año, debido a las exportaciones y a la creación de empresas filiales en el extranjero. El Centro de Inmunología Molecular es una institución que se desempeña en esta área y se dedica al estudio del cáncer y otras enfermedades, y a la producción de vacunas y medicamentos para el tratamiento de estos padecimientos.

Los biofármacos investigados y comercializados por esta empresa se basan en la fermentación continua de células animales y se producen en las plantas de fermentación del propio centro. El buen estado de los sistemas de control del equipamiento es un requisito clave para poder alcanzar los niveles de calidad requeridos en la obtención de estos biofármacos, debido a esto resulta de vital importancia lograr que estos procesos tengan un nivel alto de automatización, con sistemas capaces de controlar los distintos procesos de forma eficiente y de mantener las variables fundamentales en un fermentador, dentro de los parámetros establecidos.

En estos procesos se controlan variables como temperatura, $\mathrm{pH}$, presión, $\mathrm{O}_{2}$ disuelto, nivel en el interior del fermentador y velocidad de agitación. Estas variables deben mantenerse dentro de un intervalo de valores específicos, garantizando así las condiciones necesarias para que el cultivo celular crezca y se desarrolle adecuadamente. Además se requiere un sistema de supervisión que permita conocer el estado del proceso en cada momento, almacenar información acerca del comportamiento del mismo, y emitir alarmas en caso de que se produzcan situaciones anormales.

\section{I.1 SITUACIÓN PROBLEMA}

La Planta Piloto del CIM cuenta con una serie de fermentadores fuera de uso, las principales causas se muestran a continuación:

$\checkmark$ Problemas en el sistema de adquisición de las variables del proceso provocando fallos frecuentes en las secuencias de operación, lo que desencadenó en contaminaciones del medio de cultivo y bajos niveles de productividad.

$\checkmark$ Elevados riesgos de operación ya que algunas de las secuencias de operaciones se realizaban de forma manual, repercutiendo en la calidad del producto.

$\checkmark$ Los fallos (por operación automática) en las corridas de fermentación representaban el $87 \%$ de las fallas totales en el año 2015, hasta su descontinuación.

$\checkmark$ Repetidas asistencias técnicas por parte de los fabricantes del equipo para solucionar los problemas que iban surgiendo, y no podían dar solución por la obsolescencia de la tecnología instalada.

\section{I.2 OBJETIVOS}

Realizar la Re-Automatización del Fermentador Techfors 14L de la planta piloto del CIM, incorporar nuevos lazos de control e implementar un nuevo sistema de supervisión.

\section{I.3 ASPECTOS GENERALES DE LOS PROCESOS DE FERMENTACIÓN}

Un proceso de fermentación típico es esencialmente un proceso que se lleva a cabo en un recipiente llamado fermentador o biorreactor, mediante el cual determinados sustratos que componen el medio de cultivo son transformados por acción microbiana en metabolitos y biomasa. El microorganismo va aumentando en su concentración en el transcurso del proceso al mismo tiempo que el medio se va modificando y se forman productos nuevos como consecuencia de las actividades catabólicas y anabólicas. Los dos fenómenos crecimiento y formación de producto, tienen lugar durante el desarrollo del proceso simultáneamente o no, según los casos [1].

Resumiendo, un proceso fermentativo consiste en unir un medio de cultivo que contiene nutrientes con un microorganismo dado en un fermentador, donde se establecen condiciones experimentales adecuadas para obtener finalmente el producto deseado.

\section{I.4 DESCRIPCIÓN TECNOLÓGICA DEL PROCESO DE FERMENTACIÓN EN EL TECHFORS 14L}

Este equipo fue fabricado en el año 1994 por la compañía INFORS HT, y su función principal radicaba en la experimentación y obtención de medicamentos para ensayos clínicos, es de tipo vaso-chaqueta de 14 litros de volumen (véase figura 1), construido de acero inoxidable en su mayor parte y contaba con la incorporación de varios sensores y actuadores para la medición y el control de las variables de mayor impacto en los procesos de crecimiento celular.

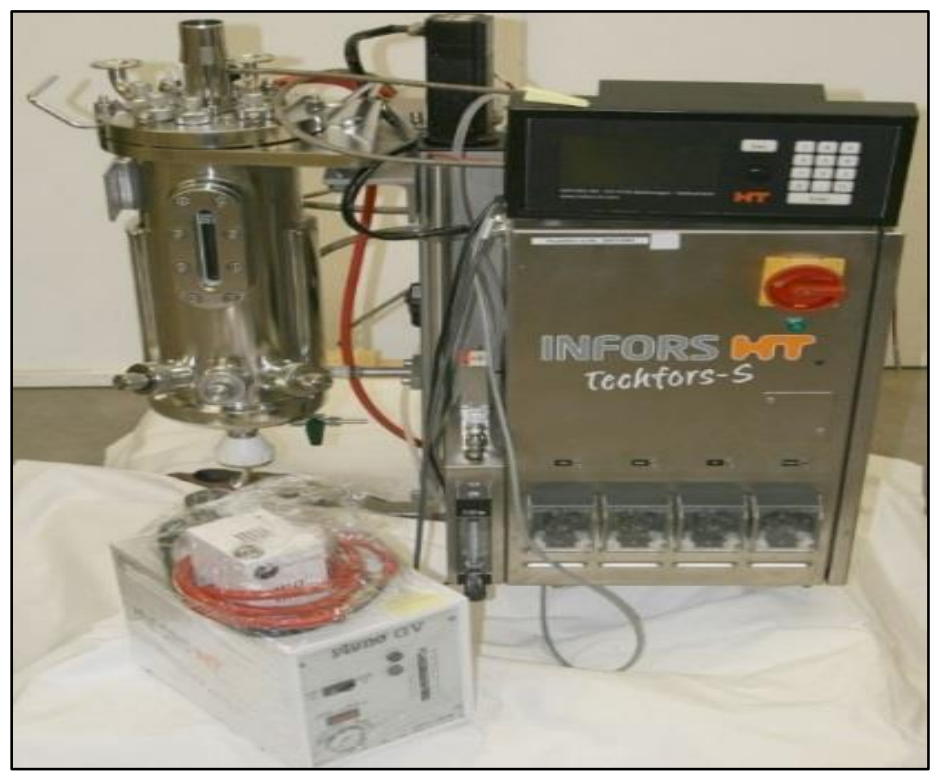

Figura 1: Fermentador Techfors 14L.

Fuente: autores, (2017).

El control de las variables del biorreactor se realizaba a través de un sistema basado en microprocesadores y tarjetas electrónicas. Este sistema estaba compuesto de un panel de control principal al que se conectan los principales sensores/transmisores (agitación, temperatura, $\mathrm{pH}$, oxígeno disuelto) y una interfaz de usuario o Human Machine Interface (HMI). Para garantizar el correcto funcionamiento del equipo, se requieren servicios críticos mostrados a continuación:

- Fuente de alimentación eléctrica: 220V VAC, 60Hz, 16A.

- Vapor limpio (esterilización): 1.5 bar.

- Entrada del agua de enfriamiento: $\geq 2.5$ bar, $20{ }^{\circ} \mathrm{C}$.

- Aire libre de aceite: 6-7 bar.

- Oxigeno $(\mathrm{O} 2)$. 
- Dióxido de Carbono (CO2).

- Nitrógeno.

El fermentador está diseñado para realizar los procesos de:

- Cleaning In Place (CIP): Este proceso se lleva a cabo con la ayuda de una bomba de recirculación que se encarga de distribuir tanto a las líneas como al vaso del fermentador las soluciones de limpieza y agua purificada para las fases de enjuague.

- Sterilization In Place (SIP): Vapor limpio $\left(121^{\circ} \mathrm{C}\right.$ durante $25 \mathrm{~min}$ ) se hace circular por todas las líneas y superficies que estén en contacto con el producto, con el objetivo de eliminar posibles contaminantes.

Con el paso del tiempo estos controles fueron fallando poco a poco, hasta que el sistema de control quedo totalmente manual operado por los tecnólogos.

\section{METODOLOGÍA}

Por los problemas mostrados anteriormente nos dimos a la tarea de diseñar, instalar, programar, configurar y poner en marcha un sistema automatizado para garantizar el funcionamiento del fermentador, garantizando el rescate de aquellos sistemas de fermentación ya caducos por el paso de los años, dotándolos nuevamente de controles eficientes y duraderos, para cumplir el objetivo necesitábamos:

- Analizar los medios técnicos de automatización existentes y proponer la instrumentación adecuada para el correcto funcionamiento del proceso.

- Diseñar, configurar y programar el control lógico secuencial, basado en Controlador Lógico Programable (PLC).

- Diseñar la aplicación para la supervisión del sistema.

Como concepto el corazón de este sistema automatizado iba a ser un PLC y teníamos como objetivo incorporar nuevos lazos de control para garantizar una automatización más integral del proceso. A continuación se muestran los lazos de control de la nueva propuesta de control del fermentador:

\section{Variables controladas en el processo:}

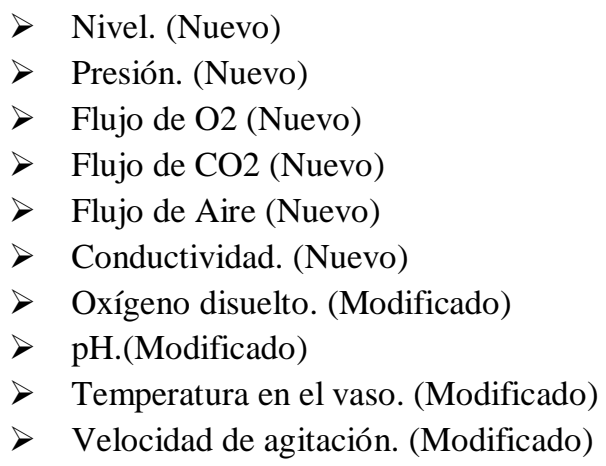

El nuevo sistema control del fermentador está basado en periferia descentralizada utilizando un bus de comunicación (Profibus) para enlazar el módulo de señales (entrada/salida). Este módulo es la interfaz entre el Controlador Lógico
Programable (PLC) y los dispositivos de campo (sensores y actuadores). La comunicación con el sistema de supervisión re realiza a través de Ethernet. A continuación se muestra la estructura del sistema de control del fermentador (véase figura 2).

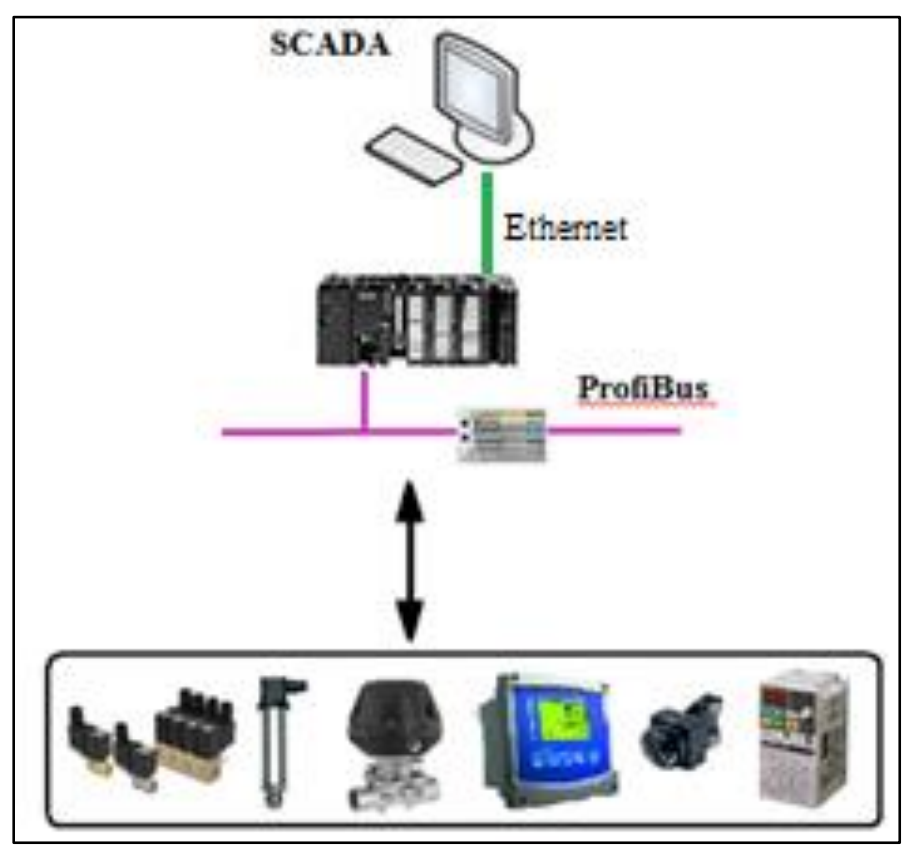

Figura 2. Sistema de control del fermentador. Fuente: autores, (2017).

\section{CONTROLADOR LÓGICO PROGRAMABLE (PLC)}

Un Autómata Programable Industrial (API) o Controlador Lógico Programable (PLC), es una máquina electrónica programable diseñada para ser utilizada en un entorno industrial (hostil), que utiliza una memoria programable para el almacenamiento interno de instrucciones orientadas al usuario, para implantar soluciones específicas tales como funciones lógicas, secuencias, temporizaciones, recuentos y funciones aritméticas, con el fin de controlar mediante entradas y salidas, digitales y analógicas diversos tipos de máquinas o procesos [2].

El autómata gobierna las señales de salidas según la lógica del programa de control previamente almacenado en una memoria en su unidad de procesamiento central $(C P U)$, a partir del estado de las señales de entrada. Este programa se inserta en el PLC a través de la unidad de programación, que permite además funciones adicionales como depuración de programas, simulación, monitorización, control del autómata y otras más.

La CPU encargada de realizar el control de todas las operaciones del fermentador y garantizar su correcto funcionamiento y seguridad es un S7-300 CPU-3152DP del fabricante SIMENS.

\section{II.2 DISEÑO DE PLANOS ELÉCTRICOS Y PI\&D}

Se realizó el nuevo diseño del PI\&D del sistema (véase figura 3), incluyendo los nuevos cambios en la instrumentación de campo, se adaptaron y acoplaron 9 nuevos sensores, 10 actuadores/válvulas de control, incorporamos una nueva bomba y una resistencia de calentamiento para el control de la temperatura y un motor agitador. 


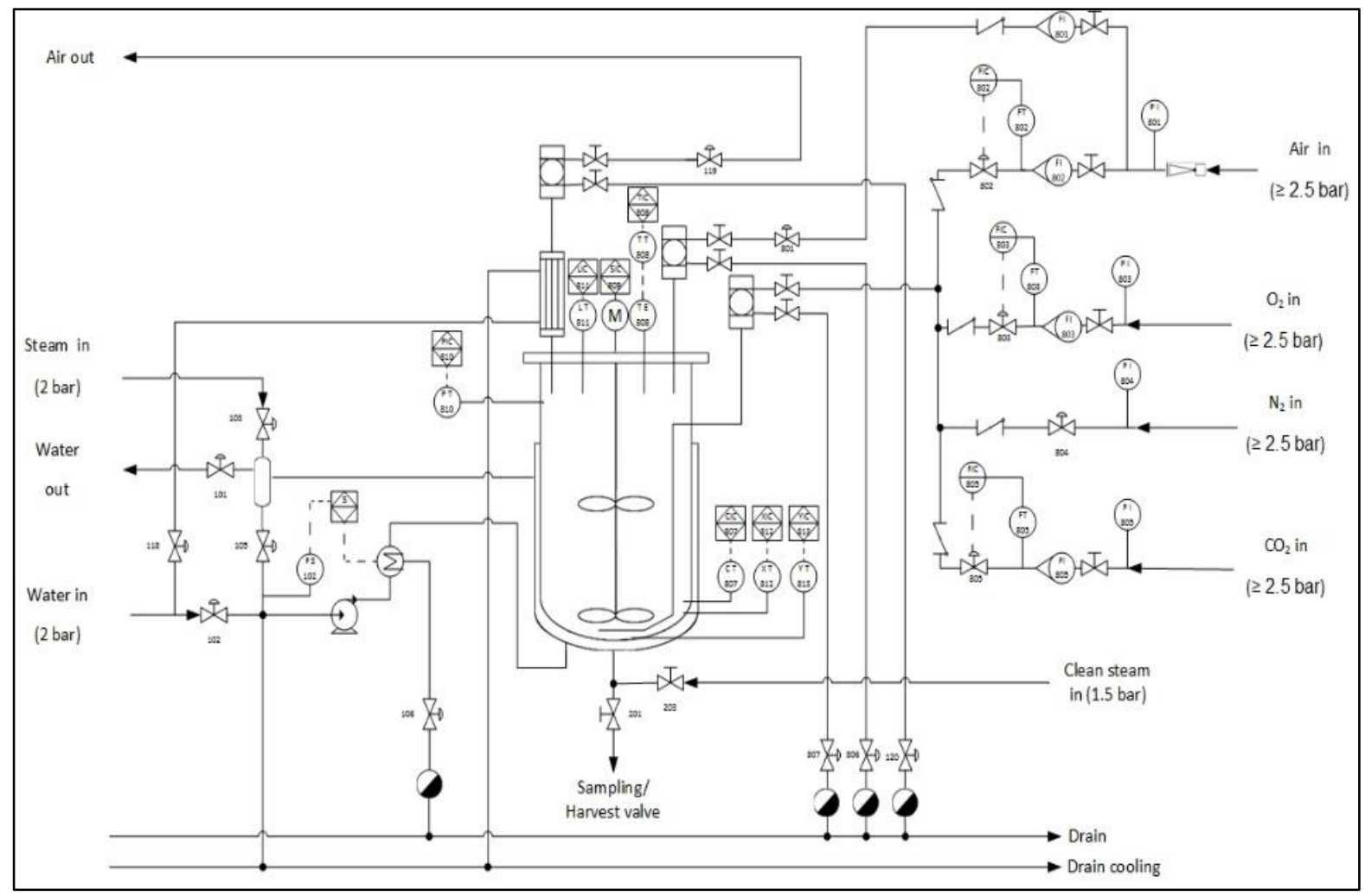

Figura 3: Nuevo diseño de PI\&D

Fuente: autores, 2017.

Se realizó el diseño de los planos eléctricos mediante el software Engeenering Base para posteriormente realizar el montaje y cableado del panel de control con los elementos de campo, en la siguiente figura se muestra un ejemplo de los planos eléctricos realizados.

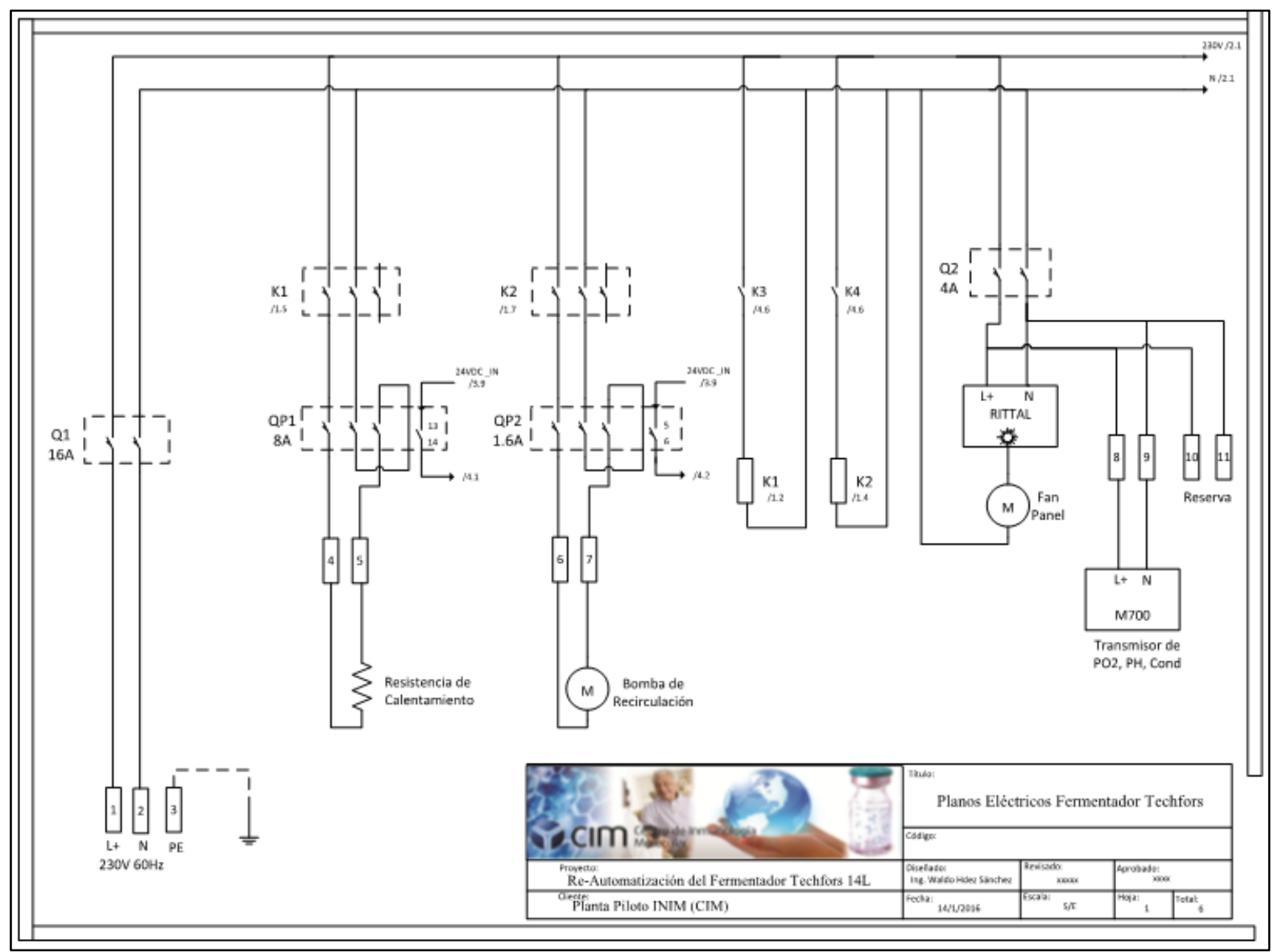

Figura 4: Diseño de planos eléctricos.

Fuente: autores, (2017). 


\section{II.3 MONTAJE DE ELEMENTOS DE CAMPO Y ADAPTACIÓN DEL NUEVO PANEL DE CONTROL}

Se realizó el montaje y adaptación de la nueva instrumentación de campo por ingenieros y técnicos mecánicos de nuestro departamento de ingeniería, a continuación se muestran imágenes del montaje.

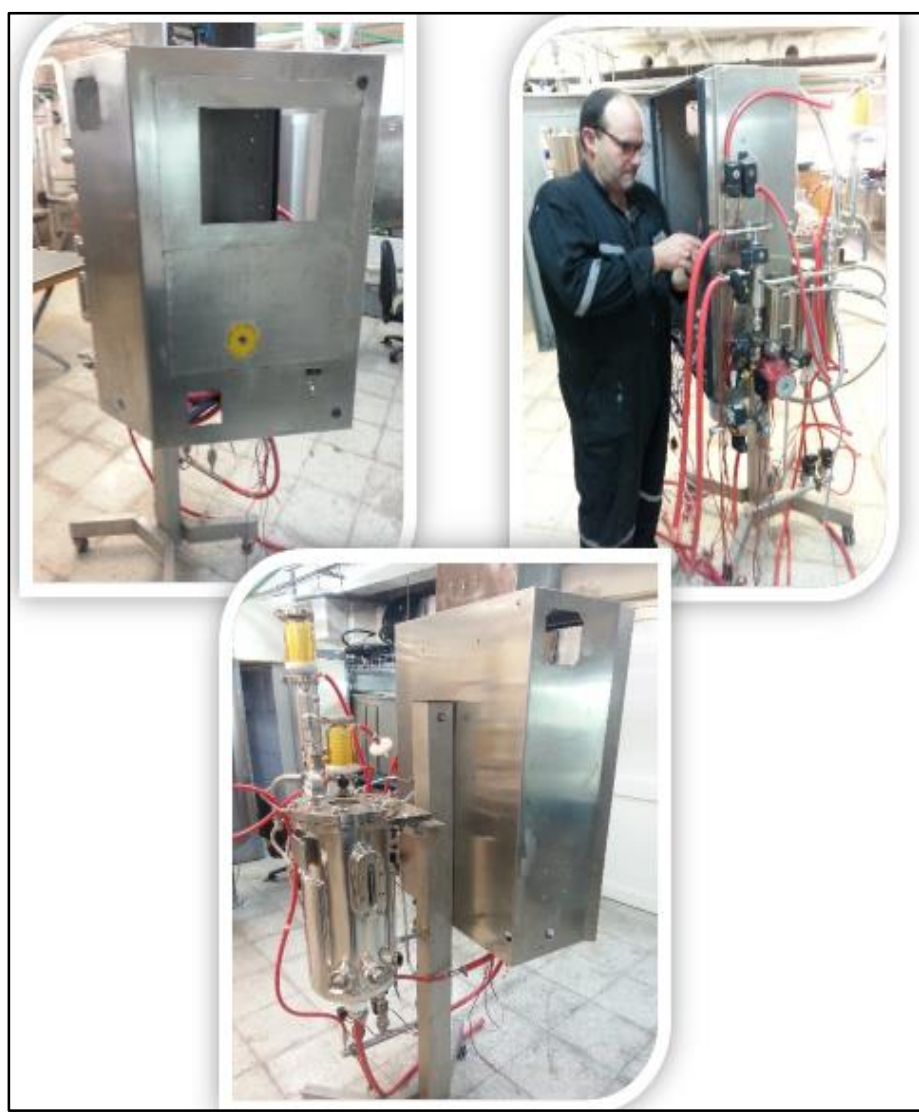

Figura 5: Montaje de instrumentación y elementos de campo. Fuente: autores, (2017).

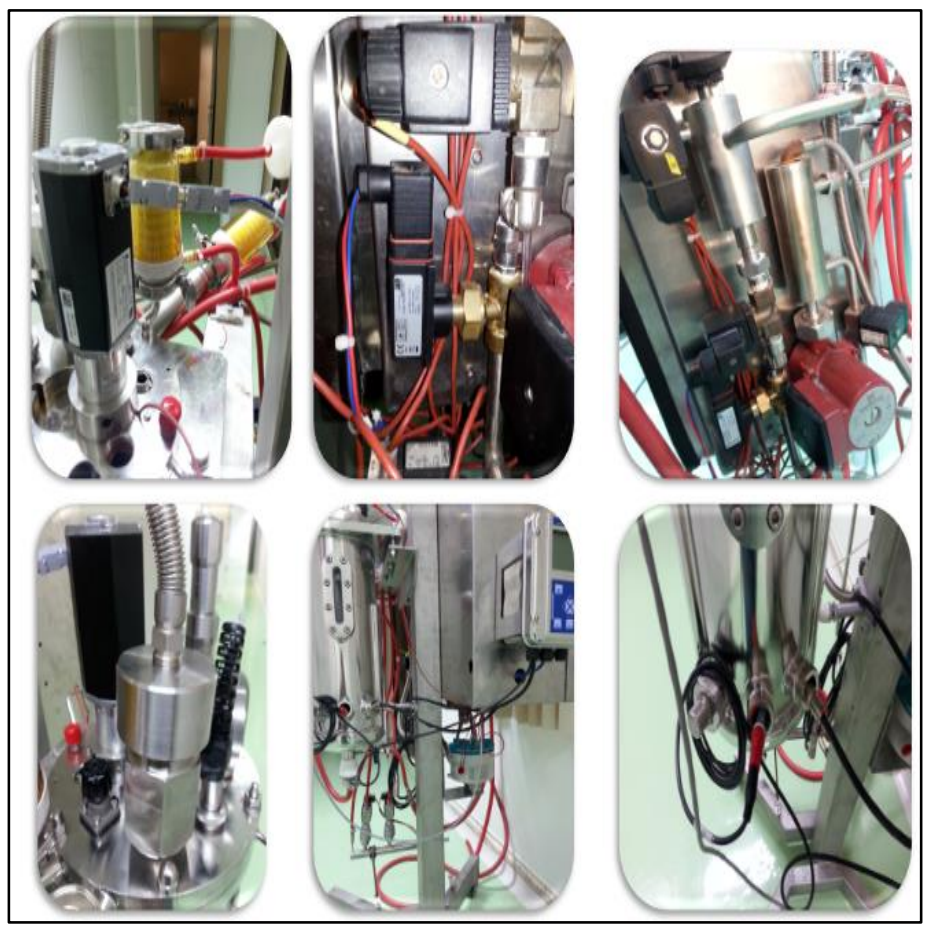

Figura 6: Montaje de sensores/transmisores y actuadores.

Fonte: autores, 2017.

\section{II.4 DISEÑO Y CABLEADO DEL PANEL DE CONTROL}

Se realizó el montaje de los elementos eléctricos y control por ingenieros y técnicos automáticos del departamento de ingeniería, a continuación se muestran imágenes del montaje. En las figuras se muestran los elementos de control:

- PLC

- Módulo de Periferia descentralizada

- HMI

- Transmisores/acondicionadores de señales

- Contactores magnéticos, relés y guardamotores

- Breakers

- Flujómetros de gases

- Fuente de alimentación

- Manómetros de presión de gases

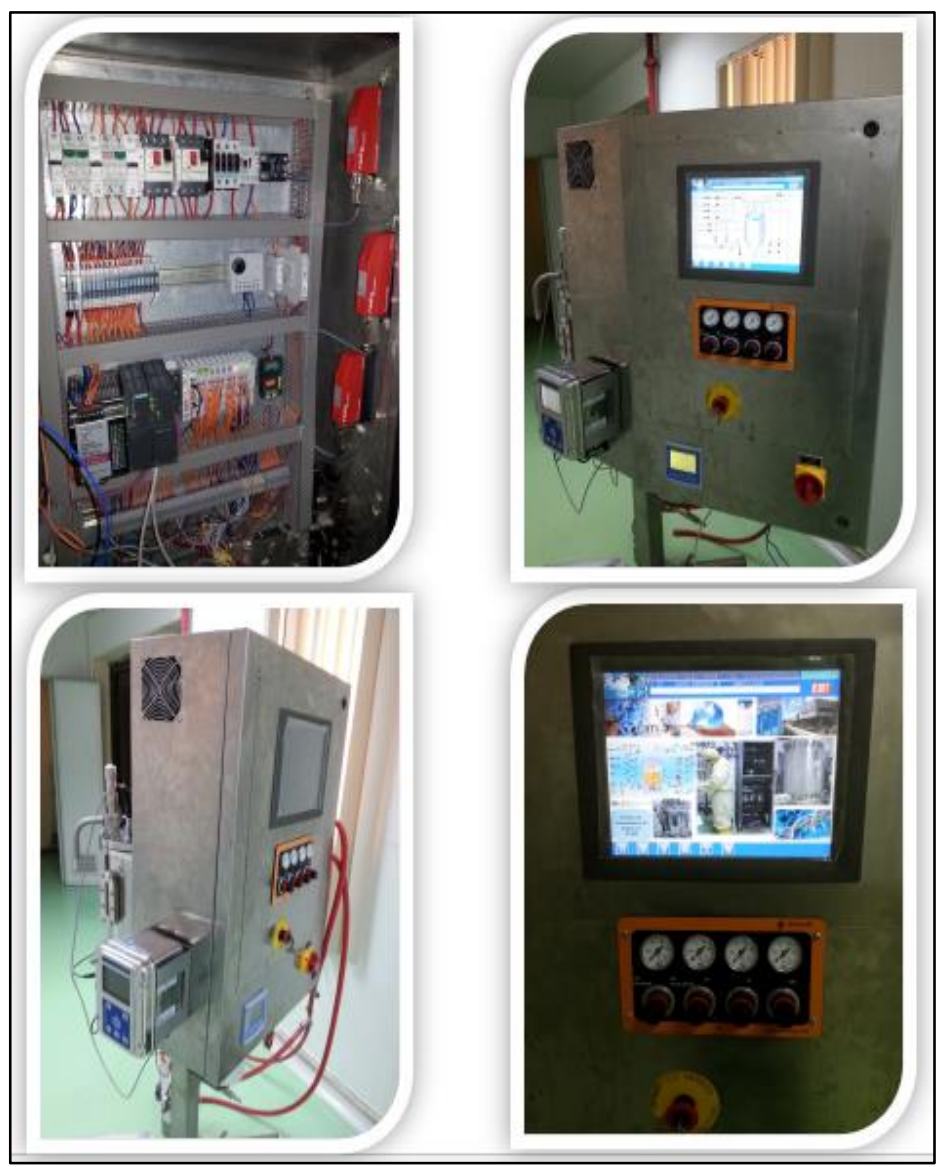

Figura 7. Diseño y cableado del panel de control.

Fuente: autores, (2017).

\section{II.4 PROGRAMACIÓN Y CONFIGURACIÓN DEL SISTEMA DE CONTROL Y SUPERVISIÓN}

El programa de control se realizó mediante STEP7 del fabricante Siemens, cuenta con alrededor de 24 bloques de funciones programadas y 15 bloques de datos, se implementaron 7 controles PID. En la siguiente figura se muestra la ventana principal de programación: 


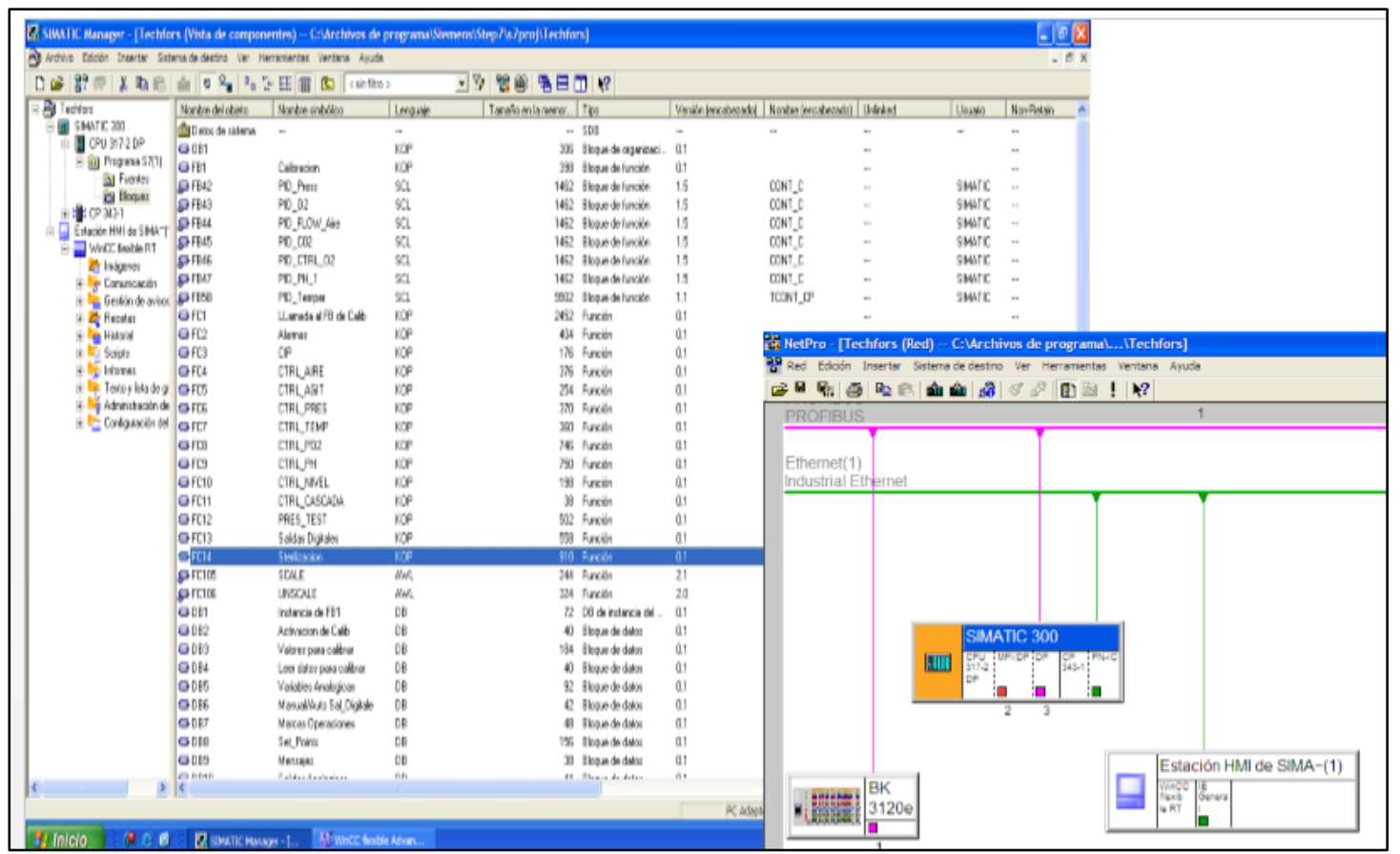

Figura 8: Bloques de programa y configuración de la red de comunicación en STEP7

Fuente: autores, (2017).

Una vez concluida la programación en el PLC, se realizó la configuración de la aplicación de supervisión mediante el software WinCC Flexible del fabricante Siemens, mediante el cual se podrá supervisar todo el sistema mediante sinópticos, almacenar las variables para el análisis de datos estadísticos, generación de alarmas, etc. En la siguiente figura se muestra la pantalla principal de este sistema.

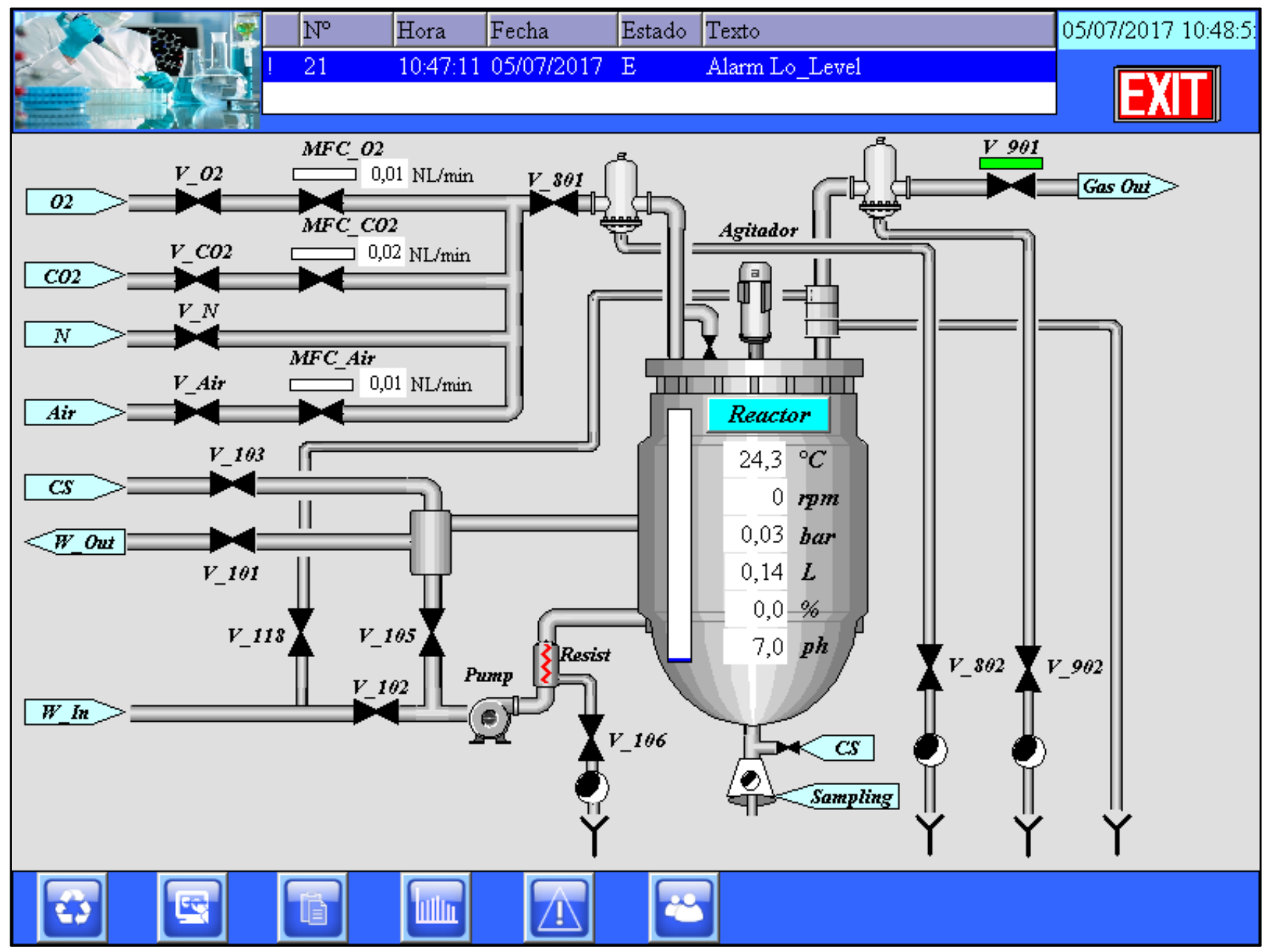

Figura 8: Bloques de programa y configuración de la red de comunicación en STEP7.

Fonte: autores, 2017. 


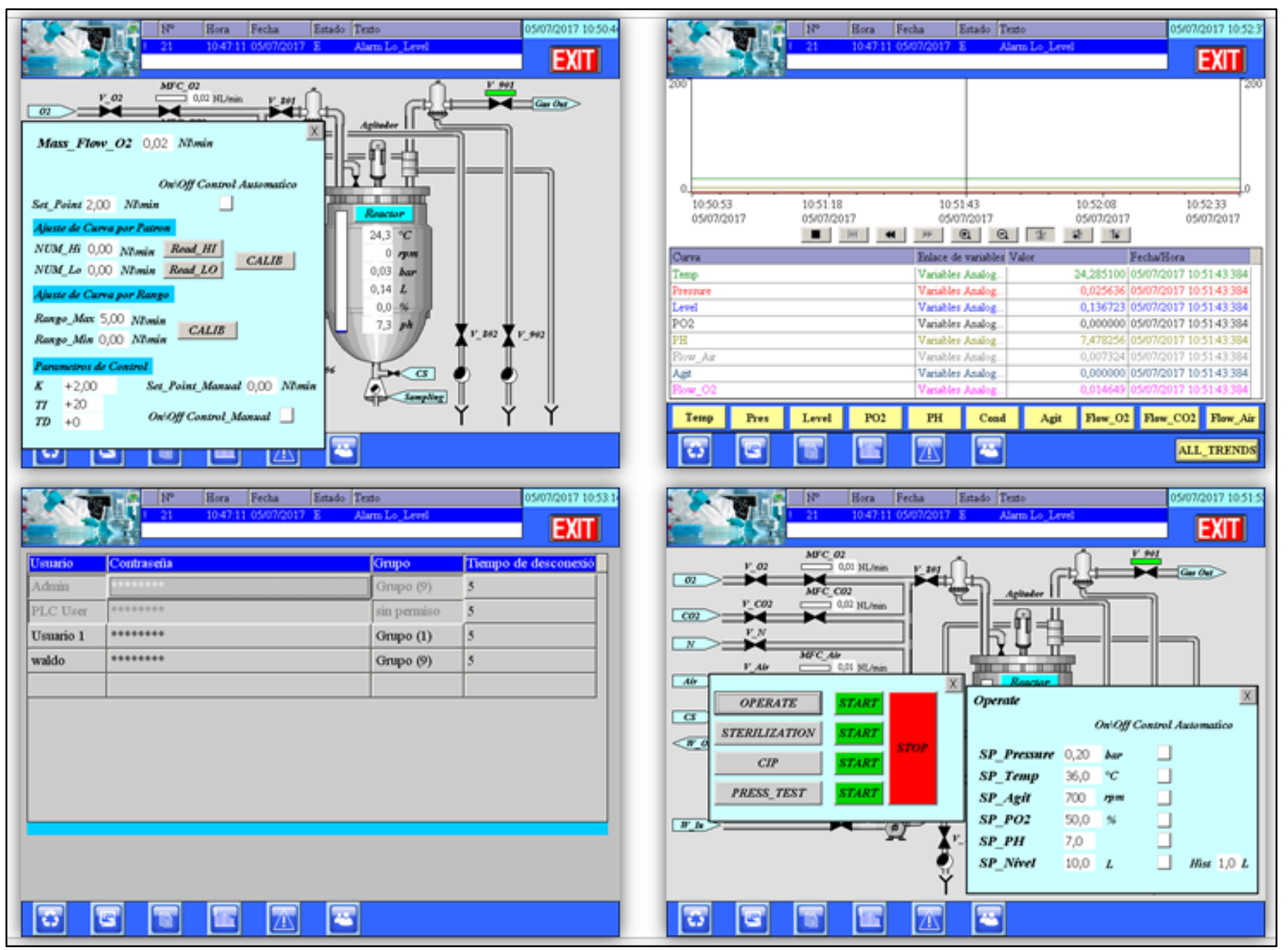

Figura 10: Pantallas de ajuste de variable analógicas, operación, históricos y configuración de usuários.

Fuente: autores, (2017).

\section{RESULTADOS Y DISCUSIÓN}

Después de concluida la etapa de montaje, programación y configuración de todo el sistema, comenzamos la puesta en marcha operativa de todos los lazos de control y secuencia de operación, obteniendo muy buenos resultados en el desempeño del sistema.

Entre los resultados más relevantes alcanzados se encuentran:

$\checkmark$ Se demuestra la capacidad que tiene nuestro departamento de ingeniería para desarrollar nuevos sistemas de control.

$\checkmark \quad$ Se diseñó e implemento un nuevo sistema de control en un fermentador, demostrando que es posible el rescate de aquellos sistemas de fermentación ya caducos por el paso de los años, dotándolos nuevamente de controles eficientes y duraderos.

$\checkmark \quad$ Se le da solución a un problema grave que presentaba este equipo impidiendo su baja técnica, contribuyendo al desarrollo de nuevos productos en la planta piloto del INIM. Se muestra a continuación un gráfico que demuestra el impacto de la incorporación de este nuevo equipo al proceso productivo e investigativo del CIM.

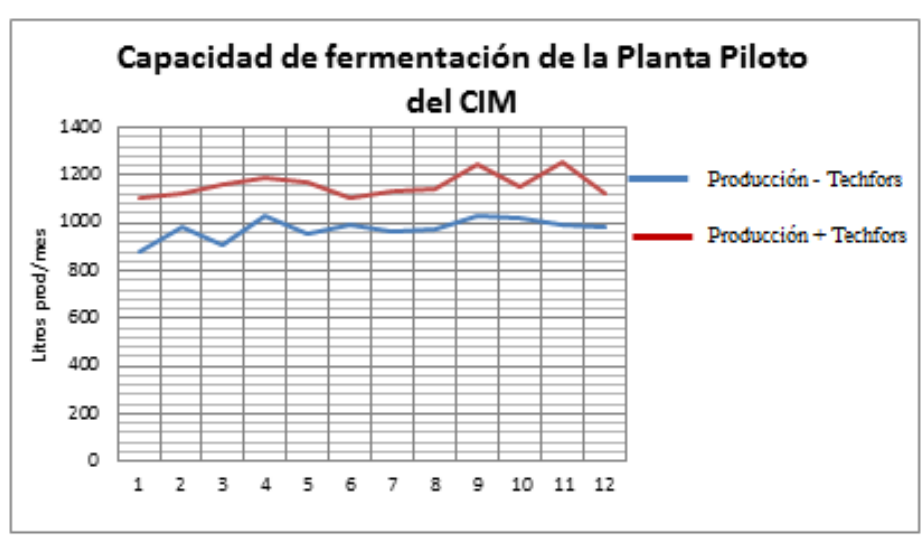

Figura 11. Grafico de obtención de producto fermentado. Fuente: autores, (2017).

$\checkmark$ Se diseñó un sistema totalmente automatizado que garantiza un elevado nivel de seguridad y autonomía en las operaciones.

Análisis Económico:

- Costo de un fermentador 14L.

aprox. 95000 euros.

- Instrumentación utilizada.

aprox. 15000 euros. 
Con la solución implementada todos estos costos son ahorrados, cerca de 80000 euros,

Además se demostró impacto que representa la incorporación de este equipo en la producción de los nuevos fitofármacos para los ensayos clínicos en pacientes.

\section{CONCLUSIONES}

- Se incorporan a este equipo controles más rigurosos y complejos para garantizar el crecimiento, adaptabilidad y desarrollo de los organismos cultivados.

- Se implementó un nuevo sistema de automatización integral de todo el fermentador, operaciones, lazos de control, procesamiento de datos, desde su software de control, hasta su sistema de supervisión.

- Se adaptó el sistema de control de este equipamiento a las necesidades propias del INIM.

- Se consolida un Know How propio del CIM en este tipo de sistemas automatizados.

\section{REFERENCIAS}

[01] Cuellas A. Biorreactores Teoría, Universidad Nacional de Quilmes, Argentina 2007.

[02] Capítulo 4, Controlador Lógico Programable PLC [Internet], Disponible
http://catarina.udlap.mx/u_dl_a/tales/documentos/lmt/maza_c_ac/ capitulo4.pdf

[03] Nuñez J. Filtración de sobrenadante de eritropoyetina producida en tanque agitado. Centro de Inmunología Molecular; 2010.

[04] Alvarado F.E. Guía de Prácticas para autómata programable basado en el S7-200 y el EM-235, Universidad de Anzuay, Ecuador, 2010.

[05] Manual Programar con STEP7 [Internet], 2006, Disponible http://cache.automation.siemens.com/dnl/jExNDU0NwAA_1865 2056_HB/S7prv54_s.pdf

[06] Siemens. Central Processing Unit CPU 314. [Internet]. 2007. Disponible en: www.siemens.com

[07] Siemens. Configurar el hardware y la comunicación con Step 7. [Internet]. 2006. Disponible en: www.siemens.com

[08] Siemens. Esquema de contactos (KOP) para S7-300 y S7400. [Internet]. 2006. Disponible en: www.siemens.com

[09] Bouzó L, Arias MA, Suárez J, Ojito E, Rodríguez E, Alvarez A, et al. Optimización de la fermentación en fermentadores de fibra hueca para la producción de biofármacos. Centro de Inmunología Molecular, La Habana, Cuba; 2002.

[10] Siemens. S7-300 CPU 31xC and CPU 31x: Technical Specifications. [Internet]. 2011. Disponible en: www.siemens.com
[11] Siemens. SIMATIC HMI, WinCC flexible 2008 SP2: Instrucciones de instalación. [Internet]. 2010. Disponible en: www.siemens.com

[12] Siemens. SIMATIC HMI, WinCC flexible 2008 SP2: Communication Part 1-2. [Internet]. 2010. Disponible en: www.siemens.com

[13] Siemens. SIMATIC HMI, WinCC flexible 2008 SP2: Runtime. [Internet]. 2010. Disponible en: www.siemens.com

[14] Siemens. SIMATIC HMI, WinCC flexible 2008 SP2: GettingStarted-Avanzado. [Internet]. 2010. Disponible en: www.siemens.com 